\title{
Migración y trabajo digno en la agricultura familiar del norte del Ecuador a lo largo del año 20ı $6^{*}$
}

Cómo citar este artículo: Eche Enríquez, David. (2017). Migración y trabajo digno en la agricultura familiar del norte de Ecuador a lo largo del año 2016. Cuadernos de Desarrollo Rural, I4(80), I-22. https://doi.org/I0.III44/Javeriana.cdrı4-80.mtda

\section{David Eche Enríquez ${ }^{\text {a }}$}

Universidad Central del Ecuador, Ecuador mdeche@uce.edu.ec

ORCID: http://orcid.org/0000-0002-7378-0066

DOI: https://doi.org/ı0.III44/Javeriana.cdri4-80.mtda

Redalyc: http://www.redalyc.org/articulo.oa?id=11756489003

Recibido: 31 Octubre 2016 I Aceptado: 22 Febrero 2017

\section{Resumen:}

Obedeciendo los parámetros postulados en la agenda de trabajo decente de la Organización Internacional del Trabajo (OIT), este estudio de investigación explora las condiciones laborales de la agricultura como los principales factores determinantes de la migración rural en tres regiones del norte del Ecuador. Con tal propósito, asume los enfoques de la agricultura familiar y de la juventud rural. Mediante métodos cualitativos y cuantitativos, se demuestra que la ausencia de trabajo decente incrementa la propensión de los agricultores a migrar y a la participación en nuevas actividades económicas, como la elaboración de artesanías y la venta de la mano de obra fuera de la finca. En tanto, la juventud rural considera que la precariedad laboral, los bajos ingresos económicos y la explotación laboral en la agricultura, así como la falta de empleo rural adecuado y digno, constituyen las principales causas de la migración y el abandono de la agricultura.

Palabras clave: condiciones laborales, agricultura familiar, desagrarización, desarrollo rural, Ecuador.

\section{Migration And Decent Work In Family Farming In Northern Ecuador Throughout 2016}

\begin{abstract}
:
\footnotetext{
a Autor de correspondencia. Correo electrónico: mdeche@uce.edu.ec
}

Following the parameters postulated in the decent work agenda of the International Labor Organization (ILO), this research study explores the working conditions of agriculture as the main determinants of rural migration in three regions of northern Ecuador. For this purpose, we assume the approaches of family farming and rural youth. We demonstrate through qualitative and quantitative methods that the absence of decent work increases the propensity of farmers to migrate and participate in new economic activities, such as the making of handicrafts and the sale of labor outside the farm. Meanwhile, rural youth consider that precarious working conditions, low economic income, and labor 
exploitation in agriculture, as well as the lack of adequate and dignified rural employment, are the main causes for migration and the abandonment of agriculture.

KeywOrds: working conditions, family farming, de-agrarization, rural development, Ecuador.

\section{Introducción}

Tanto el desarrollo de la economía global, como el de la industrialización de la agricultura, le han fallado a un gran porcentaje de la sociedad, pues han sido incapaces de proveerle trabajo decente y mejores ingresos económicos (Organización Internacional del Trabajo [OIT], 20I2). En los países subdesarrollados, este sistema de producción global genera pobreza y marginalización, al igual que migraciones de campesinos y agricultores (Hertel, 2009); además, limita particularmente las capacidades de la agricultura familiar para competir con el modo de producción agrícola industrial dominante (Barrientos, 2007). Ante la diversidad de retos, realidades y contextos en medio de los cuales se gesta la agricultura familiar, se han originado varias definiciones que buscan explorar y delimitar dichos aspectos. El 20I4 -considerado por la Organización de las Naciones Unidas para la Alimentación y la Agricultura (FAO, por sus siglas en inglés) el año internacional de la agricultura familiar- constituye un punto de referencia para la conceptualización y los lineamientos esenciales de la agricultura familiar. En este contexto, se establecen treinta y seis diferentes definiciones de varios aspectos centrales de la agricultura familiar; ejemplos de ello son mano de obra, tamaño de la unidad de producción familiar (UPA), manejo de la producción agrícola y provisión de una fuente de sustento. Una vez estos aspectos son contemplados, es posible determinar que la agricultura familiar es un medio de organización agrícola, ganadero, forestal, pastoral, pesquero y acuícola que depende netamente de la mano de obra familiar; un entorno que es manejado y operado enteramente por la familia, en un contexto en el cual existe un vínculo entre esta y la UPA, pero además se intercambian funciones económicas, sociales, ambientales y culturales (Garner y O'Campos, 2014). Cabe añadir que en su mayoría las unidades de producción agrícola del mundo tienen carácter familiar, y sus dimensiones oscilan entre $<$ I ha y 10000 ha; asimismo, las dimensiones de la UPA dependen de la disponibilidad de los recursos de los que dispone cada familia, y de las oportunidades laborales que haya afuera de la finca (Van Vliet et al., 2015).

En suma, este tipo de práctica constituye la forma más común de organización en la agricultura, inclusive en países industrializados. Asimismo, en tanto que el resultado de la intensificación de la producción agrícola no es sustentable, los países que por varias razones políticas fomentaron la agricultura familiar -particularmente asiáticos-, han logrado mayor crecimiento económico y han establecido las bases de su industrialización (Banco Mundial, 2008).

Empero, el sector agroindustrial exportador neoliberal de América Latina no genera una distribución equitativa de la riqueza (Camagnani, 2008). Además, ha estimulado la precariedad, la subcontratación y el fraude laboral en la agricultura (Gálvez, Gutiérrez, Picazzo y Osorio, 20I6). En ese escenario, junto con las determinantes socioeconómicas, la falta de trabajo decente ha incrementado la migración internacional en América Latina en los últimos años: como lo revela una medición reciente, el 5,1\% de la población de esta región reside en países como España y los Estados Unidos, lo que la ubica como la segunda diáspora más grande a nivel mundial Organización de las Naciones Unidas [ONU], 2013). Según la teoría de la nueva economía de la migración laboral (NELM, por sus siglas en inglés) centrada en los hogares, la migración es una estrategia de estos; una alternativa que busca la diversificación de ingresos familiares ante choques económicos, así como ante la falta de apoyo financiero y 
económico gubernamental (Taylor, 1999). Cabe añadir que el fenómeno mencionado se acrecienta en la actualidad, dados los nuevos eventos climatológicos (Kaenzig y Piguet, 20I6).

Adicionalmente, la agricultura constituye todavía un sector fundamental que contribuye al crecimiento económico de la mayoría de países latinoamericanos, y se caracteriza por ser predominantemente de pequeña escala y familiar (FAO, 2016). Asimismo, depende de la disponibilidad de mano de obra local para su continuidad.

En ese escenario, las limitadas opciones que tiene la agricultura familiar para la diversificación del sustento del hogar conduce a los agricultores a la búsqueda de actividades no agrícolas -es decir, al abandono de la actividad económica-; fomenta la migración hacia los centros urbanos (FAO, 2016; Gálvez et al., 2016), y termina por afectar el desarrollo de las regiones rurales, principalmente por la pérdida de la fuerza laboral agrícola (Bilsborrow, 2002; Gray y Bilsborrow, 20I4; Wiggins, 2016).

En el sentido descrito, la creciente migración de los jóvenes capacitados y emprendedores se convierte en un factor de riesgo notable para el desarrollo rural y, en particular, para el desarrollo de la agricultura familiar (Sili, Fachelli y Meiller, 2017); un aspecto que, aunado al cambio de actividad económica, conduce eventualmente a un proceso de desagrarización del campo (Bernstein, 20I2). Subsecuentemente, las precarias condiciones existentes en el sector agrícola de pequeña escala y familiar hacen que la juventud que aún permanece en las zonas rurales no considere que este sector es una fuente de desarrollo; por tanto, sus integrantes buscan oportunidades de estudio en zonas urbanas, convirtiendo a algunos pueblos rurales en pueblos dormitorio (FAO, 20I4a). Tanto la migración del campo a la ciudad, como el abandono de la actividad agrícola, tienen una estrecha relación con la seguridad alimentaria de las sociedades a mediano y largo plazo (Programa Mundial de Alimentación, 2017). En consecuencia, se acentúa todavía más la necesidad de que el trabajo decente agrícola y rural pleno y productivo se postule como meta de desarrollo (Filmer y Fox, 20I4; FAO, 20I4a).

Asimismo, el marco de trabajo de este enfoque abarca los principios de la teoría del desarrollo humano y del desarrollo como libertad de Amartya Sen (1999); base en la cual se establece que el desarrollo de una región (sociedad) debe centrarse en el otorgamiento de bienestares y libertades humanas. Cabe enfatizar que, en ese mismo escenario, estas permiten expandir el abanico de opciones que tienen los individuos para el progreso, para así asegurar una mayor inclusión social.

En este contexto, el trabajo decente es importante para el desarrollo humano y sustentable; en tanto, mediante la OIT se "promueven oportunidades para que los hombres y las mujeres puedan conseguir un trabajo decente y productivo en condiciones de libertad, equidad, seguridad y dignidad humana” (OIT, 1999, p. 2). Posteriores conceptualizaciones describen el trabajo decente como:

la oportunidad de acceder a un empleo productivo que genere un ingreso justo, la seguridad en el lugar de trabajo y la protección social para las familias, mejores perspectivas de desarrollo personal e integración social, libertad para que los individuos expresen sus opiniones, se organicen y participen en las decisiones que afectan sus vidas, y la igualdad de oportunidades y trato para todos, mujeres y hombres. (OIT, 2007, p. 4)

Estas aspiraciones laborales se agrupan en cuatro pilares: empleo, protección social, derechos y dialogo social (Barrientos, 2007; Ghai, 2003; FAO, 20I2; OIT, 1999, 2007, 20I2). En ese sentido, dichos aspectos sirven de guía para la construcción de los resultados en este trabajo de investigación. Además, la Organización de las Naciones Unidas (ONU) y la Organización Internacional del Trabajo (OIT), así como diversos centros de investigación especializados y múltiples agencias internacionales dirigidas hacia el desarrollo, consideran que el fomento y la generación de empleo pleno y productivo, al igual que la aplicación de los estándares del trabajo decente, son necesarios para la erradicación de la pobreza y para la consecución de los Objetivos de Desarrollo Sostenible (ODS) 2015-2030 (OIT, 2012; FAO, 2014a).

En particular, el objetivo de los ODS 2015-2030 se centra en considerar el trabajo decente una alternativa para el crecimiento económico. Para la obtención de tal propósito, las nuevas políticas y programas se enfocan en incrementar la productividad laboral; mejorar el acceso a servicios financieros; reducir el desempleo entre 
la población joven, y eliminar la explotación infantil (ONU, 20I6). En consonancia, la OIT propone al trabajo decente como una alternativa idónea para avanzar hacia una mayor igualdad; promover condiciones mínimas para que todos los trabajadores puedan cubrir sus necesidades básicas; fomentar el desarrollo rural, y disminuir las tasas de migración (OIT, 20I2).

En el contexto ecuatoriano, el Plan Nacional para el Buen Vivir 2013-20I7 propone en el marco del noveno objetivo "garantizar el trabajo digno en todas sus formas"; añade además que, para la obtención de este objetivo, se deben "generar trabajos en condiciones dignas, buscar el pleno empleo priorizando a grupos históricamente excluidos, reducir el trabajo informal y garantizar el cumplimiento de los derechos laborales” (Senplades, 2013, p. 75).

Sin embargo, las iniciativas que se han emprendido para el análisis de las condiciones de trabajo decente en alguna rama del sector económico del país son limitadas. En ese sentido, Viteri (2010) arguye que, aunque se han hecho esfuerzos para el establecimiento de un modelo de trabajo decente, las limitaciones económicas y las diferencias del mercado de trabajo no han permitido continuar avanzando en la materia. En un contexto en el que el avance en materia de trabajo decente es pausado, con el paso del tiempo, las deficiencias se han hecho más evidentes, ante todo en el sector de la agricultura. Junto con los trabajadores agrícolas, una porción significativa de los agricultores se encuentra en condiciones de inseguridad y precariedad laboral (OIT, 2006; FAO, 2014a).

En concordancia con lo descrito, el presente estudio se perfila como una contribución que, con base en información empírica, se propone revivir el debate académico organizado en torno al desarrollo agrícola y rural. En ese marco, se contemplará el trabajo decente como una alternativa idónea para la reducción de la pobreza y la migración; asimismo, se atiende la necesidad de generar conciencia política y social de un tema crucial pero descuidado.

\section{Tipo de metodología}

La presente investigación explora las condiciones laborales actuales de la agricultura familiar del norte de Ecuador, en el marco de la agenda de trabajo decente definida por la OIT. En ese contexto, se propone determinar los principales aspectos socioeconómicos y demográficos de los cultivadores que desarrollan sus actividades como parte de la agricultura familiar, al igual que examinar las percepciones de estos en el campo, y las de los estudiantes en colegios agrícolas. En tal escenario, por medio de análisis descriptivos e inferenciales, se analiza las determinantes; asimismo, con base en los enfoques de la agricultura familiar y la juventud rural, se explican los nexos que existen entre las condiciones laborales precarias (ausencia de trabajo decente), la pobreza, la migración y el abandono de la actividad económica, y la desagrarización del campo como resultado final. Adicionalmente, la identificación empírica del vínculo existente entre la desagrarización y las condiciones laborales del sector agrícola, permitirá formular recomendaciones para una política agraria coherente que integre variables sociales; propuesta postulada con miras a la reducción de problemas como la migración rural-urbana, los cambios demográficos y culturales, y la precariedad laboral del sector agrícola y rural.

La información primaria fue recolectada entre los años 2015 y 2016, mediante la implementación de dos tipos de encuestas estructuradas de pregunta cerrada; asimismo, se practicaron entrevistas a informantes claves, autoridades locales y expertos localizados en tres provincias diferentes de las tres regiones geográficas del Ecuador. Las encuestas del primer tipo fueron diseñadas con la finalidad de recolectar información al nivel de la agricultura familiar, y fueron aplicadas a la cabeza del hogar: esta herramienta ha permitido recoger información sociodemográfica, económica y agrícola, al igual que datos relativos a las condiciones laborales y las percepciones migratorias. En concordancia, se determinó una muestra total para los 3 sitios de estudio de 443 encuestas, de las 
cuales 159 se obtuvieron en la provincia de Manabí, región Costa; 196 en la provincia de Imbabura, región Sierra, y 88 en la provincia de Francisco de Orellana, en la Amazonía (ver Figura i).

En tanto, un segundo grupo de cuestionarios fue diseñado con el objetivo de recolectar información primaria. Dicha medición fue desarrollada con la finalidad de determinar la percepción que los jóvenes de instituciones educativas agropecuarias tienen de su futura participación en el sector agrícola (continuidad-abandono); de su posible emigración, y de las condiciones laborales que ofrece la agricultura (trabajo decente).

En la región Costa, las encuestas se aplicaron en el Colegio Técnico Agropecuario Unión y Progreso, ubicado en la Bramadora de la parroquia Manga del Cura de la provincia de Manabí; una zona que ha sido delimitada recientemente. En la región Sierra, las mediciones fueron llevadas a cabo en el Colegio Técnico Agropecuario Fernando Chávez Reyes del cantón Otavalo, en la provincia de Imbabura. En la región amazónica, se desarrolló la investigación en la Unidad Educativa Fiscomisional Técnico Agropecuario Padre Miguel Gamboa, que está localizada en el cantón San Sebastián del Coca, en la provincia de Francisco de Orellana. De estas instituciones, se encuestó un total de 366 estudiantes pertenecientes a los dos últimos años de bachillerato.

Este proyecto de investigación es la primera fase de un análisis nacional en el cual se tienen en cuenta factores como la ubicación geográfica (zona norte), la división regional (Costa, Sierra, Oriente) y la importancia agrícola del cantón. Asimismo, son contemplados aspectos como el tamaño de cada unidad de producción agrícola (UPA), las tasas de migración y la presencia de instituciones educativas agropecuarias.

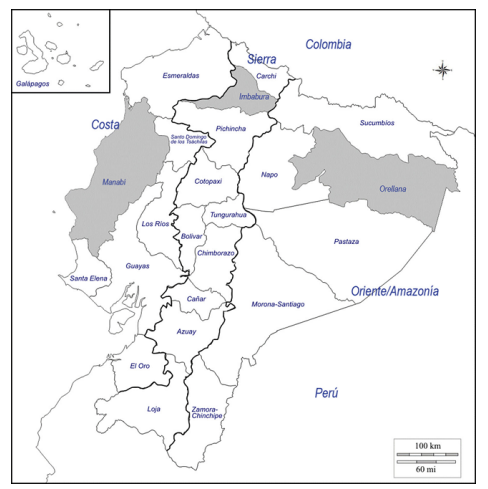

FIGURA 1

UBICACIÓN DE LOS SITIOS DE ESTUDIO POR PROVINCIA Y REGIÓN

FUENTE: ELABORACIÓN PROPIA

Adicionalmente, con la presentación descriptiva de las variables que componen las dimensiones del trabajo decente, se realiza un análisis de los coeficientes (log-odds) de regresión logística, para determinar los efectos de algunas determinantes socioeconómicas sobre la migración en la agricultura familiar. En consonancia, los indicadores utilizados para este caso de estudio están clasificados de acuerdo con los cuatro pilares del trabajo decente: el empleo, la seguridad social, los derechos de los trabajadores, y el diálogo social.

\section{Resultados y discusión}

Mediante un análisis descriptivo de las principales variables sociodemográficas que caracterizan a las y los jefes de hogar, se determina que existe una diferencia entre las regiones con respecto a la edad, el tamaño de la UPA y el número de personas que componen el hogar. Asimismo, entre las diversas actividades agropecuarias que son desarrolladas, se nota que la agricultura familiar es la actividad económica predominante en las tres regiones; en tanto, en lo que respecta a la tenencia de la UPA, esta se caracteriza por ser propia. 


\section{Empleo}

Los indicadores laborales utilizados para el análisis de esta dimensión provienen de las oportunidades laborales expresadas en términos del porcentaje de la población económicamente activa (PEA) que se ocupa en el sector primario, al igual que de tasas de empleo y desempleo. Según la última Encuesta Nacional de Empleo, Desempleo y Subempleo (Instituto Nacional de Estadística y Censos [INEC], 20I4) la tasa de ocupados plenos (TOP) muestra un mejor panorama de las oportunidades de trabajo, en tanto que el empleo y el desempleo son contemplados como valores separados. Adicionalmente, la variable TOP provee información sobre la proporción de personas en edad de trabajo que están comprometidas con alguna actividad de producción de bienes y servicios. Además, cubre todas las categorías de los trabajadores, y puede demostrar si la proporción de personas que trabajan en la economía se encuentra en incremento o decrecimiento (Ghai, 2003). En ese escenario, la tabla I demuestra las diferencias que existen entre las tres regiones en lo que respecta a esta variable; además, cuando se toma en consideración la tasa de desempleo (TdD), es posible notar que dicho valor es inferior en el sector rural a nivel nacional, mientras que la región Costa es el lugar en donde existe mayor desempleo rural. Sin embargo, la población en pobreza extrema (PePE) es mayor en la región de la Amazonía, en comparación a las otras regiones.

TABLA 1

DESCRIPCIÓN DE VARIABLES DE EMPLEO Y POBREZA POR REGIONES

\begin{tabular}{c|l|rr|rr|rr|rr}
\multicolumn{2}{c|}{} & \multicolumn{2}{c|}{ Costa } & \multicolumn{2}{c|}{ Sierra } & \multicolumn{2}{c|}{ Amazonía } & \multicolumn{2}{c}{ Nacional } \\
\cline { 3 - 9 } \multicolumn{2}{c|}{} & Urbana & Rural & Urbana & Rural & Urbana & Rural & Urbana & Rural \\
\hline \multirow{6}{*}{$\%$} & PEA & 42,9 & 36,4 & 46,5 & 46,7 & 42,0 & 37,5 & 44,3 & 41,7 \\
& TOP & 47,6 & 53,1 & 59,9 & 66,5 & 52,3 & 61,1 & 52,7 & 60,8 \\
& TdS & 45,8 & 74,3 & 34,5 & 72,8 & 41,9 & 72,1 & 41,1 & 73,1 \\
& TdD & 6,4 & 3,3 & 4,7 & 1,7 & 5,5 & 2,5 & 5,7 & 2,3 \\
& PePI* & 20,9 & 40,0 & 17,2 & 33,0 & 20,6 & 57,9 & 15, & 38,2 \\
& PePE* & 5,8 & 15,3 & 5,8 & 14,8 & 9,0 & 38,4 & 4,5 & 17,6
\end{tabular}

Fuente: elaboración propia con base en INEC (2016)

Nota: población económicamente activa (PEA); tasa de ocupados plenos (TOP); tasa de subempleo (TdS); tasa de desempleo (TdD); población de pobreza por ingresos (PePI)*; población en pobreza extrema (PePE)*

\section{Ingreso económico}

Los datos empíricos demuestran que existen otras diferencias particulares entre las tres regiones. En ese sentido, la tabla 2 detalla que el ingreso agrícola (IA) generado por los hogares es mayor en la Costa (USD \$390) que en las regiones de la Sierra (USD \$267) y la Amazonía (USD \$160). Al comparar estos ingresos con el salario mínimo unificado (SMU) de USD \$384 para el 20r8, se determina que solo los agricultores en la Costa reciben un ingreso mayor al SMU (I,5\%), en contraste con la región de la Sierra (69,5\%) y la Amazónica (41,6\%). No obstante, estos ingresos no reflejan el tamaño de la UPA. En la región Amazónica, el promedio de la UPA es de veintiún hectáreas, y los agricultores generan los ingresos más bajos. Para manifestar estas diferencias, se divide el IA por el tamaño de la UPA; en una escala de cero (O) a uno (I), de modo que se considera que uno (I) es el máximo valor (ver Tabla 2), los agricultores en la región de la Amazonía tienen la mayor brecha entre ingresos económicos y recursos naturales.

En ese escenario, las diferencias del tamaño de la tierra se derivan del minúsculo efecto han tenido las diferentes reformas agrarias y la ley de tierras. En tanto, la inequidad de la concentración de la tierra es determinada mediante 
el coeficiente de Gini, de modo que uno (I) corresponde a la mayor concentración de la tierra, y cero (o) a lo contrario. Según dichos marcos, este valor era de o,86 en 1954; de 0,85 en 1974, y de o,80 en el año 2000, fecha del último censo agropecuario. Lo anterior demuestra que la concentración de la tierra en el Ecuador es de la más altas de América Latina, si se compara con Cuba $(0,2 \mathrm{I})$ y Nicaragua $(0,32)$, países que han realizado profundas restructuraciones agrarias. En tanto, al nivel de regiones del Ecuador, la concentración de la tierra es mayor en la Sierra (Gini o,810) que en la Costa (Gini o,753) (Brassel, Ruiz y Zapata, 2008).

Cabe añadir, más específicamente, que la Sierra se caracteriza por fenómenos de acelerada fragmentación de la propiedad rural; por una eventual disminución del sostenimiento de la agricultura familiar; por altas tasas de emigración rural (Bilsborrow, 2002; Gray, 2009; Jokisch, 2002); por la degradación de los recursos naturales -en particular, el agua y el suelo-; por los cambios culturales que enfrentan los pueblos indígenas, y por el remplazo de las actividades agrícolas por las artesanales.

TABLA 2

PrinCIPAles CARACTERÍSTICAS SOCIOECONÓMICAS

\begin{tabular}{l|r|r|r|r|r|r}
\cline { 2 - 7 } \multicolumn{1}{c|}{ Región } & $\begin{array}{c}\text { Ingreso } \\
\text { económico } \\
\text { (USD) }\end{array}$ & $\begin{array}{c}\text { Sin ingreso } \\
\text { económico } \\
(\%)\end{array}$ & $\begin{array}{c}\text { Tamaño } \\
\text { UPA (m2) }\end{array}$ & $\begin{array}{c}\text { IE vs } \\
\text { TUPA }\end{array}$ & $\begin{array}{c}\text { Edad } \\
\text { (años) }\end{array}$ & $\begin{array}{c}\text { Tamaño del } \\
\text { hogar }\end{array}$ \\
\hline Costa & 390,7 & 1,8 & 75551,7 & 0,005 & 50 & 4,3 \\
\hline Sierra & 172,0 & 30,6 & 3127,1 & 0,055 & 45 & 5,5 \\
\hline Amazonía & 160,5 & 1,1 & 209979,5 & 0,000 & 42 & 6,2 \\
\hline Promedio total & 272,6 & 14,4 & 70211,8 & 0,003 & 45,6 & 5,3 \\
\hline
\end{tabular}

Fuente: elaboración propia

\section{Apreciación del ingreso económico y de la rentabilidad de la actividad económica}

Posteriormente, se analizó la apreciación que hace el agricultor del ingreso económico. En ese sentido, la tabla 3 detalla que casi la mitad de la población considera que el ingreso agrícola es regular, y un tercio lo considera pobre, en comparación con el SMU. En un análisis más profundo, se determinó que siete de cada diez campesinos consideran que la agricultura es una actividad económica no rentable. Estas condiciones forman parte de los principales motivos para que cerca de la mitad de los agricultores iniciaran una nueva actividad económica secundaria que les generara mejores réditos económicos. 
TABLA 3

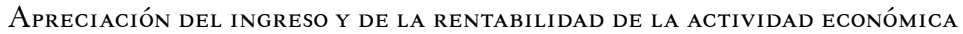

\begin{tabular}{c|l|r|r|r|r}
\cline { 3 - 6 } \multicolumn{2}{c|}{} & $\begin{array}{c}\text { Costa } \\
\mathbf{N = 1 9 6}\end{array}$ & $\begin{array}{c}\text { Sierra } \\
\mathbf{N}=\mathbf{1 5 9}\end{array}$ & $\begin{array}{c}\text { Amazonía } \\
\mathbf{N = 8 8}\end{array}$ & $\begin{array}{c}\text { Total } \\
\mathbf{N}=\mathbf{4 4 3}\end{array}$ \\
\hline \multirow{4}{*}{$\begin{array}{c}\text { Apreciación } \\
\text { del ingreso }\end{array}$} & Muy bueno & 1,9 & 1,0 & 0,9 & 1,3 \\
& Bueno & 7,7 & 13,1 & 11,7 & 11,0 \\
& Aceptable & 12,5 & 21,4 & 12,6 & 16,8 \\
& Regular & $\mathbf{4 9 , 7}$ & $\mathbf{3 8 , 4}$ & $\mathbf{4 9 , 5}$ & $\mathbf{4 4 , 3}$ \\
& Pobre & 28,0 & 25,9 & 25,2 & 26,5 \\
\hline La actividad & Sí & 29,9 & 24,5 & 35,1 & 28,3 \\
económica es & No & $\mathbf{7 0 , 0}$ & $\mathbf{7 5 , 4}$ & $\mathbf{6 4 , 8}$ & $\mathbf{7 1 , 6}$ \\
rentable & & $\mathbf{4 0 , 8}$ & $\mathbf{5 0 , 0}$ & $\mathbf{3 6 , 3}$ & $\mathbf{4 4 , 0}$ \\
\hline Con actividad & Sín & 59,1 & $\mathbf{5 0 , 0}$ & 63,6 & 55,9 \\
económica & No & & & &
\end{tabular}

Fuente: elaboración propia

\section{Indicadores del desarrollo humano}

Estos indicadores componen las temáticas de educación, salud e ingresos económicos. Como lo demuestra la Enemdu (INEC, 20I6), a nivel nacional, en el sector rural, la diferencia en la incidencia de la pobreza por ingresos (PePI) y la población en pobreza extrema (PePE) es, respectivamente, dos y cuatro veces mayor, en comparación con el área urbana (ver Tabla I). En concordancia, se evidencia que un alto porcentaje de la población de la región amazónica se encuentra en condiciones de pobreza extrema rural. En lo que refiere al nivel de educación, existe una diferencia notoria en el porcentaje de personas calificadas como iletradas, de modo que el mayor porcentaje se sitúa en la región de la Sierra con 31,3\%; en tanto, el 8,2\% se localiza en la Costa, y el menor porcentaje se sitúa en la Amazonía, con el 3,4\%.

\section{Derechos laborales}

\section{Explotación laboral}

La principal preocupación de la agricultura y, en particular, de la de carácter familiar, es la de la explotación infantil, en especial cuando se da condiciones de abuso y de discriminación en el trabajo. La OIT (20I7) estima que existen alrededor de 152 millones de niños -cuyas edades oscilan entre 5 y 17 años- sujetos al trabajo infantil, mientras que cerca de 40 millones de personas son víctimas de la esclavitud moderna; una gran parte estos se ubica en la agricultura familiar o en empresas familiares (OIT, 20I6).

La participación de la mano de obra familiar es fundamental para el desarrollo de la agricultura de pequeña y mediana escala, pues además de ser una herencia cultural, facilita la continuidad de esta práctica a lo largo del tiempo y el espacio. Adentro de la agricultura familiar, tanto la participación de los hijos de los agricultores en las actividades agrícolas, como su remuneración, son variables que contribuirán a entender la explotación infantil, 
y su relación con la migración de espacios rurales a contextos urbanos. En los tres sitios de estudio de la región amazónica, se determinó que más de la mitad de hogares cuenta con la mano de obra de los hijos (ver Figura 2).

Sin embargo, con el fin de retribuir dicha participación e incentivar su continuidad, estas prácticas agrícolas pueden ser remuneradas. Es así como, en la agricultura familiar de la región Costa, la mayoría de los agricultores remunera económicamente la contribución laboral de sus hijos. En contraste, y en similar proporción, en la agricultura familiar de la Sierra no se remunera el trabajo infantil y familiar. Aunque un alto porcentaje de los agricultores de las tres regiones envíe a sus hijos a alguna unidad académica de enseñanza primaria, secundaria y terciaria, la población en edad estudiantil que no recibe educación adecuada, se integra a la mano de obra; sirve así como una medida complementaria de la incorporación de dicha población a la agricultura (Kucera, 200I).

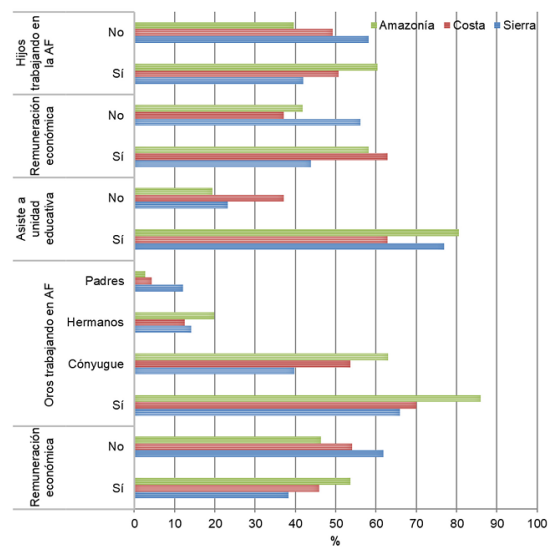

FIGURA 2

Trabajo Agrícola FAMILIAR, REMUNERACIÓN ECONÓMICA Y ASISTENCIA ESCOLAR Nota: representación de los porcentajes desagregados de las variables sobre la participación de los miembros del hogar.

FUENTE: ELABORACIÓN PROPIA

La participación de otros miembros de la familia en las diversas actividades agrícolas es importante para la continuidad y el desarrollo de la agricultura familiar. En la Amazonía también existe una mayor contribución del cónyuge a las actividades agrícolas y, en menor proporción, resulta esencial la participación de los hermanos y los padres. En ese plano, su dependencia de la fuerza de trabajo familiar hace que este tipo de agricultura sea la más vulnerable ante la pérdida de la mano de obra local, a causa de la migración de los espacios rurales a las esferas urbanas.

En ese contexto, la jornada laboral es una variable la cual permite concluir que el número de horas de trabajo que establece el agricultor para sí mismo, al igual que la extensión de la jornada laboral, están enmarcadas en alguna forma de autoexplotación laboral. En ese sentido, la figura 3 demuestra que la jornada laboral es irregular en las tres regiones: un fenómeno notorio, particularmente, en la Sierra, en donde la jornada laboral del agricultor es mayor a las ocho horas establecidas. En la región Amazónica, en contraste, un mayor porcentaje de los agricultores cumple con la jornada laboral establecida, así como también con un menor número de horas. Cabe señalar que la diferencia de la jornada laboral entre las regiones está ligada al desarrollo económico y humano, al igual que a las características ambientales y culturales particulares, así como a la disponibilidad de recursos naturales. 


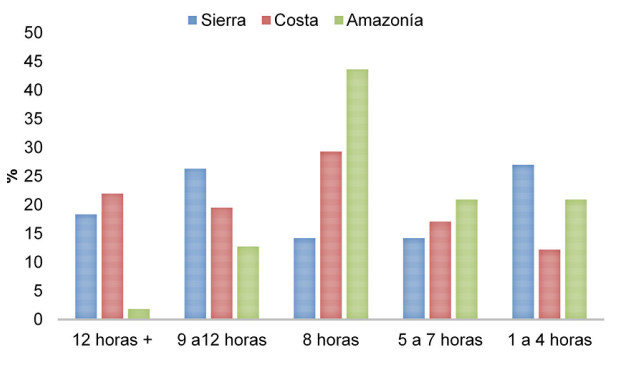

FIGURA 3

DESCRIPCIÓN DE LA JORNADA LABORAL AGRÍCOLA

Nota: los indicadores son parte de las dimensiones de empleo y derechos de los trabajadores.

FuENTE: ELABORACIÓN PROPIA

En este escenario, la discriminación y la explotación en el trabajo remiten a la ausencia de oportunidades, al igual que a la inequidad en torno al aspecto dual de lo social ante lo laboral. En tanto, la tabla 4 muestra el porcentaje total de la población agrícola que considera que ha sufrido de explotación laboral; una circunstancia debida a los bajos e injustos salarios que existen en el sector agrícola.

TABLA 4

EXPLOTACIÓN LABORAL Y TIPOS DE EXPLOTACIÓN

\begin{tabular}{|c|c|c|c|c|c|}
\hline & & $\begin{array}{c}\text { Costa } \\
\mathrm{N}=196 \\
\end{array}$ & $\begin{array}{r}\text { Sierra } \\
\mathbf{N}=159 \\
\end{array}$ & $\begin{array}{c}\text { Amazonía } \\
\mathbf{N}=\mathbf{8 8}\end{array}$ & $\begin{array}{c}\text { Total } \\
\mathrm{N}=\mathbf{4 4 3} \\
\end{array}$ \\
\hline \multirow{2}{*}{$\begin{array}{l}\text { Explotación } \\
\text { laboral }\end{array}$} & Sí & 74,0 & 62,9 & 73,8 & 68,8 \\
\hline & No & 25,0 & 37,0 & 26,1 & 31,1 \\
\hline \multirow{6}{*}{$\begin{array}{l}\text { Tipo de } \\
\text { explotación } \\
\text { laboral }\end{array}$} & Salarios injustos & 73,8 & 81,8 & 74,3 & $\overline{77,1}$ \\
\hline & Labores sin contratos de trabajo & 9,2 & 4,9 & 12,2 & 8,1 \\
\hline & Horas de trabajo excesivas & 4,9 & 4,9 & 1,2 & 4,0 \\
\hline & Labores sin seguridad social & 1,8 & 3,3 & 0,0 & 1,9 \\
\hline & Trabajo extra no remunerado & 9,2 & 2,7 & 4,8 & 5,7 \\
\hline & Otro & 1,2 & 2,2 & 7,3 & 3,1 \\
\hline
\end{tabular}

Fuente: elaboración propia

En tanto, la figura 4 demuestra que un elevado porcentaje de los agricultores considera que las condiciones laborales de las tres regiones son regulares y pobres; una percepción predominante en las regiones Amazónica y de la Costa. Sin embargo, también existe un porcentaje menor que considera que las condiciones laborales son buenas y aceptables. 


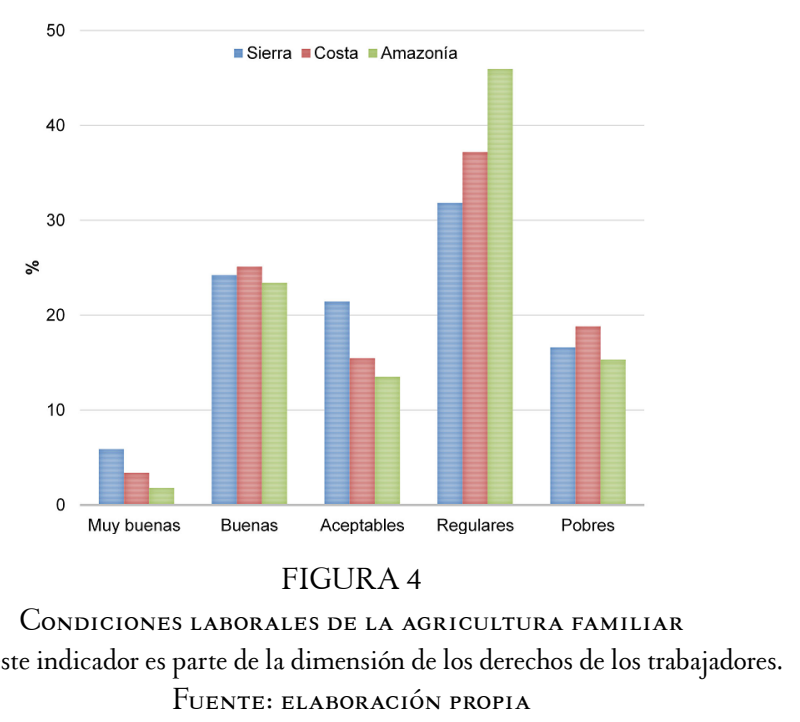

Adicionalmente, con el fin de diferenciar las brechas de género existentes, se analizan la oportunidad de empleo por género y la participación de la mujer en la agricultura familiar. Entre las tres regiones de estudio, se determina que el $47 \%$ de los hogares tiene a la mujer como jefe de hogar; en tanto, esta participación es menor en las regiones de la Costa y la Amazonía, con el 30\% y 26\%, respectivamente. Con miras a futuros análisis estadísticos, la variable del género se pondera, para reducir el peso de la brecha que dicho aspecto ejerce sobre los resultados.

\section{Seguridad social}

Es importante señalar que la seguridad social no está presente en el sector agrícola. Esta provee protección a los trabajadores ante eventualidades, y además sirve para cubrir las necesidades urgentes de las personas (OIT, 20I2). En los países desarrollados, con la seguridad social se busca asistir a poblaciones vulnerables mediante una importante gama de programas de asistencia social. En contraste, la situación es muy diferente en los países subdesarrollados, dado el pequeño porcentaje de empleados asalariados que se vinculan allí a la economía formal en comparación a la población total: efectivamente, en la mayoría de estos países, la seguridad social falla (Ghai, 2003). En Ecuador, la población rural cuenta con el Seguro Social Campesino, disposición gubernamental que otorga programas de salud integrales y pensiones de jubilación e invalidez al trabajador (Instituto Ecuatoriano de Seguridad Social, 2006). Sin embargo, como lo evidencia la tabla 5 -que se ocupa de las perspectivas regionales del asunto-, nueve de cada diez agricultores en la Sierra, y siete de cada diez en la Amazonía, no disponen del Seguro Social Campesino para la protección ante los peligros laborales causados por factores como el manejo de agroquímicos, herramientas y maquinaria agrícola. En ese sentido, hay marcadas diferencias con la Costa, en donde siete de cada diez agricultores se encuentran asegurados socialmente. No obstante, para más de la mitad de la población, la agricultura no es considerada una actividad económica segura laboralmente y libre de peligros. 
TABLA 5

SEguros médicos y Riesgos LABORALES EN LA AGRicultura

\begin{tabular}{|c|c|c|c|c|c|}
\hline & & $\begin{array}{c}\text { Costa } \\
N=196\end{array}$ & $\begin{array}{c}\text { Sierra } \\
\mathbf{N}=\mathbf{1 5 9}\end{array}$ & $\begin{array}{c}\text { Amazonía } \\
\mathbf{N}=\mathbf{8 8}\end{array}$ & Total \\
\hline \multirow{3}{*}{$\begin{array}{l}\text { La agricultura es } \\
\text { segura y libre de } \\
\text { peligros }\end{array}$} & Sí & 46,3 & 44,2 & 41,4 & 44,4 \\
\hline & No & 53,6 & 55,7 & 58,5 & 55,5 \\
\hline & Sí & 69,0 & 10,0 & 29,7 & 33,7 \\
\hline Seguro médico & No & 30,9 & 89,9 & 70,2 & 66,2 \\
\hline
\end{tabular}

Fuente: elaboración propia

\section{Diálogo social}

Uno de los aspectos más importantes de esta dimensión del trabajo decente es la democracia económica sustentada mediante la participación en actividades o emprendimientos enmarcados en la economía formal e informal. Sin embargo, las medidas de la democracia económica no son simples (Ghai, 2003). Para responder esta interrogante, se utiliza la variable proxy que corrobora si el agricultor realiza una actividad económica secundaria y que clasifica dicha actividad según su tipo. En ese sentido, los valores expresados en la tabla 6 resaltan la alta participación de los agricultores en actividades económicas secundarias; especialmente, estos caracterizan el involucramiento de los campesinos en el mercado laboral agrícola como jornaleros.

Sin embargo, los indicadores descritos hasta el momento apuntan hacia una labor agrícola que no se enmarca en la agenda del trabajo decente. Esta situación, en conjunto con las expuestas, hace que dos de cada diez agricultores consideren abandonar la agricultura y que, en similar proporción, estimen que la migración constituye una alternativa de diversificación económica. En un análisis por regiones, un mayor porcentaje de los agricultores en la Sierra considera la posibilidad de migrar -acción entendida como el abandono del campo-, mientras que en la Costa los agricultores optan por el abandono de la actividad agrícola y el inicio de una nueva actividad económica.

Hay cinco razones principales que influyen en la decisión de abandonar la agricultura. Estas son (i) la ausencia de apoyos económicos y técnicos al sector por parte del gobierno, (ii) los bajos ingresos económicos, (iii) la baja productividad, (iv) la degradación de los suelos, y (v) la migración de los trabajadores agrícolas, particularmente en la Sierra. En tanto, los bajos indicadores socioeconómicos, las condiciones laborales y las largas jornadas laborales descritas hasta el momento se convierten en aspectos determinantes para las decisiones en materia de emigración y abandono de la actividad agrícola.

Además, se evidencia el eventual abandono de la actividad agrícola y la transformación del agricultor en jornalero agrario temporal, así como la disminución de la agricultura familiar. Finalmente, en los tres sitios de estudio, la mitad de los agricultores consideran estar obligados a ejercer su actividad económica, o básicamente a ser agricultores familiares de pequeña escala. Lo anterior demuestra que el carácter cultural y hereditario de la agricultura generacional familiar eventualmente se degrada, a expensas del subdesarrollo y las precarias condiciones laborales en que se desenvuelven las labores; circunstancias que hacen que la agricultura sea menos atractiva para la mano de obra familiar. 


\begin{tabular}{|c|c|c|c|c|c|}
\hline & & $\begin{array}{c}\text { Costa } \\
N=196\end{array}$ & $\begin{array}{c}\text { Sierra } \\
N=159\end{array}$ & $\begin{array}{c}\text { Amazonía } \\
\mathbf{N}=\mathbf{8 8}\end{array}$ & Total \\
\hline \multirow{6}{*}{$\begin{array}{l}\text { Actividad } \\
\text { económica } \\
\text { secundaria }\end{array}$} & Sí & 40,88 & 50,0 & 36,3 & 55,9 \\
\hline & Jornalero & 13,8 & 10,2 & 12,5 & 11,9 \\
\hline & Chofer & 6,9 & 0,5 & 0,0 & 2,7 \\
\hline & Albañil & 1,2 & 7,6 & 2,2 & 4,2 \\
\hline & Negocio propio / comerciante & 8,8 & 5,1 & 5,6 & 6,7 \\
\hline & Otro & 10,0 & 26,0 & 15,9 & 18,2 \\
\hline \multirow{2}{*}{ Piensa emigrar } & Sí & 11,1 & 24,5 & 15,3 & 18,2 \\
\hline & No & 88,8 & 75,4 & 84,6 & 81,7 \\
\hline \multirow{2}{*}{$\begin{array}{l}\text { Abandonar la } \\
\text { agricultura }\end{array}$} & Sí & 22,7 & 16,9 & 8,1 & 17,3 \\
\hline & No & 73,9 & 69,5 & 90,9 & 74,9 \\
\hline \multirow{9}{*}{$\begin{array}{l}\text { Razones para el } \\
\text { abandono de la } \\
\text { agricultura }\end{array}$} & Falta de apoyos económicos & 21,2 & 42,8 & 66,6 & 35,2 \\
\hline & Suelos pobres / degradados & 8,5 & 14,2 & 0,0 & 10,4 \\
\hline & Bajo rendimiento agrícola & 12,7 & 10,2 & 0,0 & 10,4 \\
\hline & Bajos ingresos económicos & 27,6 & 8,1 & 22,2 & 18,1 \\
\hline & $\begin{array}{l}\text { Contaminación (del suelo, } \\
\text { agua o aire) }\end{array}$ & 10,6 & 0,0 & 0,0 & 4,7 \\
\hline & $\begin{array}{l}\text { Bajos precios de los productos } \\
\text { agrícolas }\end{array}$ & 10,6 & 6,1 & 11,1 & 8,5 \\
\hline & $\begin{array}{l}\text { Migración de trabajadores } \\
\text { locales }\end{array}$ & 0,0 & 8,1 & 0,0 & 3,8 \\
\hline & Por la edad & 8,5 & 6,1 & 0,0 & 6,6 \\
\hline & Otro & 0,0 & 4,0 & 0,0 & 1,9 \\
\hline
\end{tabular}

Fuente: elaboración propia

\section{Determinación del trabajo decente sobre la migración}

Con el fin de responder a la hipótesis central del presente estudio, que busca determinar estadísticamente la influencia de las variables del trabajo decente sobre la consideración de emigrar y las percepciones de los agricultores, se analizan dichos aspectos de forma detallada mediante una regresión logística. Para este estudio, se considera si el agricultor piensa emigrar como la variable dependiente (explicativa), la cual se pone a prueba con diecinueve variables independientes (predictoras), para determinar significativamente cuál de ellas influye sobre las probabilidades de que la migración ocurra. La variable explicativa fue codificada como $X_{i}=o$ si no piensa emigrar, y $X_{i}=I$ si piensa emigrar; asimismo, del total de encuestas $(n=443)$, cuatrocientas doce encuestas fueron seleccionadas por sus características dicotómicas $(X=I /$ Sí; $X=2 /$ No). 
Por las características de la variable dependiente, el análisis estadístico inferencial se realiza mediante el modelo logit. Cabe señalar que la regresión de este tipo busca determinar la probabilidad de que el proceso de emigración se incremente o disminuya, según las variables predictoras.

Para el modelo de la regresión logística, la ecuación es:

$$
\operatorname{logit}=\left(\pi_{i}\right)=\log \frac{\pi_{i}}{1-\pi_{i}}=\beta_{0}+\beta_{1} X_{1 i}+\ldots \beta_{j} X_{j i}
$$

Dentro de la regresión logística, los parámetros del modelo logit son estimados mediante el método de máxima verosimilitud; es decir, se seleccionan los coeficientes que hacen la observación más verosímil o probable.

En ese contexto, la ecuación teórica para el modelo es la siguiente:

$$
\begin{aligned}
& \operatorname{logit}=\left(\pi_{i}\right)=\log \frac{\pi_{i}}{1-\pi_{i}}=\beta_{0}+\beta_{1}(L O C)+\beta_{2}(G E N)+\beta_{3}(E D A D)+4(E D U C)+ \\
& \beta_{5}\left(T A M_{\text {HOGAR }}\right)+\beta_{6}\left(T E N_{\text {TIERRA }}\right)+\beta_{7}(\text { AREA })+\beta_{8}(T E A)+\beta_{9}(I N G)+\beta_{10}\left(\text { APRE } E_{\text {ING }}\right)+ \\
& \beta_{11}(M H C A)+\beta_{12}\left(I N G_{A D E C}\right)+\beta_{13}\left(C O N D_{L A B O R}\right)+\beta_{14}(J O R N A D A)+\beta_{15}\left(E X P_{L A B O R}\right)+ \\
& \beta_{16}\left(S E G_{M E D}\right)+\beta_{17}(\text { DISCRIM })+\beta_{18}(\text { HTGAR })+\beta_{19\left(A B A N D_{A G R}\right)}
\end{aligned}
$$

Donde $L O C$ es la localidad, GEN el género, EDAD la edad, EDUC la educación, TAM $M_{H O G A R}$ el tamaño del hogar, TEN $N_{\text {TIERRA }}$ la tenencia de la tierra, $A R E A$ el área, TEA el tiempo ejerciendo la agricultura, ING el ingreso económico, $A G R_{R E N T}$ que la agricultura sea rentable, $M H C A$ los miembros del hogar en la agricultura, $I N G_{A D E C}$ el ingreso económico adecuado con relación al trabajo, $C O N D_{\text {LABOR }}$ las condiciones laborales en la agricultura, JORNADA la jornada laboral, EXP ${ }_{L A B O R}$ la explotación laboral agrícola, $S E G_{M E D}$ el seguro médico, DISCRIM la discriminación en la agricultura, HTAGR el trabajo de los hijos en la agricultura, y $A B A N D_{A G R}$ el abandono de la actividad agrícola.

En tanto, los resultados de la regresión expuestos en las tablas 7 y 8 indican que el modelo es significativo $(\chi=$ 58,852; $\mathrm{p}<=0,05)$. Además, la bondad de ajuste del modelo es determinada por el test de Hosmer y Lemeshow como no significativo $(\chi=8,632 ; \mathrm{p}<=0,05)$, lo cual indica que no existe evidencia de un ajuste pobre.

TABLA 7

Prueba Omnibus de modelo de coeficientes

\begin{tabular}{l|l|rrr}
\hline & & Chi cuadrado & df & Sig. \\
\hline Paso 1 & Paso & 58,852 & 19 & $0,000^{* * *}$ \\
& Bloque & 58,852 & 19 & $0,000^{* * *}$ \\
& Modelo & 58,852 & 19 & $0,000^{* * *}$ \\
&
\end{tabular}

Fuente: elaboración propia Nota: ${ }^{* * *}$ significativo; $p=<0,00 \mathrm{I}$ 
TABLA 8

Test de Hosmer y Lemeshow

\begin{tabular}{l|rrr}
\hline & Chi cuadrado & df & Sig. \\
\hline Paso 1 & 8,632 & 8 & 0,374 \\
\hline
\end{tabular}

Fuente: elaboración propia

Nota: no significativo; $p=>0,05$

En ese escenario, los resultados de los coeficientes (log-odds) detallados en la tabla 9 demuestran que las siguientes variables predictoras son significativas. Se alude a (i) la localidad $(\beta=-0,753 ; p<=0,05)$; en tanto, para (ii) la edad $(\beta=-0,026 ; p<=0,05)$, por cada año que se incremente en la edad del agricultor, se espera una reducción de 0,026 en los coeficientes que nivelan sus posibilidades de emigración, si se mantienen todas las variables independientes constantes. Por su parte, (iii) la educación $(\beta=-0,014 ; p<=0,05)$ denota que en la medida en que la educación del jefe de hogar sea mayor, menor será su interés por emigrar. En lo que atañe (iv) al tamaño del hogar $(\beta=0,098 ; \mathrm{p}<=0,05)$, se estima que en el grado en que el número de miembros en el hogar sea mayor, el coeficiente que define la posibilidad de pensar en emigrar se incrementa en 0,098 . En lo que respecta a (v) la tenencia de la tierra $(\beta=-0,327 ; \mathrm{p}<=0, \mathrm{I})$, esta demuestra que los agricultores dueños de las UPAs consideran menos la opción a emigrar, en comparación con los que implementan UPAs rentadas. En cuanto a (vi) la rentabilidad de la agricultura $(\beta=-0,566 ; \mathrm{p}<=0,05)$, este valor expresa que quienes aún consideran la agricultura como una actividad económica rentable, piensan menos en la opción de emigrar. Sobre la temática de (vii) los miembros del hogar que se involucran con la agricultura (MHCA) $(\beta=0,488 ; p<=0, \mathrm{I})$, es importante señalar que, según este valor, en los casos en los que los agricultores manejan la UPA sin la participación de algún miembro del hogar, se incrementa el coeficiente de la emigración en 0,488 . En lo que alude a (viii) la explotación laboral $(\beta=0,467 ; \mathrm{p}<=0, \mathrm{I})$, este valor demuestra que un aumento de la explotación laboral incrementa la necesidad de emigrar. Asimismo, son reveladores los datos correspondientes a (ix) los hijos que trabajan en la agricultura $(H T A G R)(\beta=-0,473 ; \mathrm{p}<=0, \mathrm{I})$, pues significan que la ausencia de los hijos en la práctica agraria reduce el interés en emigrar. En tanto, el rubro que corresponde $(\mathrm{x})$ al abandono de la actividad agrícola $(\beta=0,625 ; \mathrm{p}<=0,05)$ demuestra que un incremento de la percepción o de la idea de abandonar la actividad agrícola incrementa los coeficientes de la posibilidad de emigrar en 0,625 , en un escenario en el que todas las variables predictoras se mantengan constantes. 
TABLA 9

RESULTADO DE LAS VARIABLES PREDICTORAS USADAS EN LA REGRESIÓN

\begin{tabular}{|c|c|c|c|c|c|}
\hline & & $\mathrm{B}$ & Wald & Sig. & $\operatorname{Exp}(\mathrm{B})$ \\
\hline \multirow[t]{20}{*}{ Paso 1} & $L O C$ & $-0,75$ & 7,28 & $0,007^{* *}$ & 0,47 \\
\hline & GEN & $-0,25$ & 1,04 & 0,308 & 0,77 \\
\hline & $E D A D$ & $-0,02$ & 6,91 & $0,009 * *$ & 0,97 \\
\hline & EDUC & $-0,21$ & 6,05 & $0,014^{* *}$ & 0,80 \\
\hline & $T A M_{H O G A R}$ & 0,09 & 4,33 & $0,037^{* \star}$ & 1,10 \\
\hline & $T E N_{\text {TIERRA }}$ & $-0,32$ & 2,91 & $0,088^{\star}$ & 0,72 \\
\hline & $A R E A$ & 0,14 & 0,63 & 0,427 & 1,15 \\
\hline & TEA & $-0,26$ & 0,46 & 0,496 & 0,76 \\
\hline & ING & 0,10 & 0,58 & 0,445 & 1,11 \\
\hline & $A G R_{R E N T}$ & $-0,56$ & 4,25 & $0,039 * *$ & 0,56 \\
\hline & $M H C A$ & 0,48 & 3,42 & $0,064^{\star}$ & 1,63 \\
\hline & $I N G_{A D E C}$ & 0,13 & 0,20 & 0,654 & 1,14 \\
\hline & $C O N D_{L A B O R}$ & $-0,05$ & 0,246 & 0,620 & 0,94 \\
\hline & JORNADA & 0,04 & 0,20 & 0,653 & 1,04 \\
\hline & $E X P_{L A B O R}$ & 0,46 & 2,80 & $0,094^{\star}$ & 1,59 \\
\hline & $S E G_{M F n}$ & 0,40 & 1,95 & 0,162 & 1,49 \\
\hline & DISCRIM & 0,04 & 0,02 & 0,868 & 1,04 \\
\hline & HTAGR & $-0,47$ & 3,15 & $0,076^{*}$ & 0,62 \\
\hline & $A B A N D_{A G R}$ & 0,62 & 4,80 & $0,028^{* \star}$ & 1,86 \\
\hline & Constant & 0,47 & 0,17 & 0,674 & 1,61 \\
\hline
\end{tabular}

Fuente: elaboración propia

Nota: variable(s) ingresadas en el paso i: LOC, GEN, EDAD, EDUC, TAM HOGAR, TEN TIERRA, AREA,

TEA, ING, AGR RENT $, M H C A, I N G_{A D E C}, C O N D_{L A B O R}, J O R N A D A, E X P_{L A B O R}, S E G_{M E D}$,

DISCRIM, HTAGR, ABAND $A G R \cdot{ }^{*}$ significativo al ०,I; ** significativo al ०,०5; ${ }^{* * *}$ significativo al ०,०0I.

Esta investigación es reforzada por la información empírica obtenida de estudiantes que están matriculados en instituciones educativas agrícolas; cotejo realizado con el fin de explorar, desde la perspectiva de la juventud rural, las percepciones de la migración, así como sus motivaciones, y nociones esenciales en materia de explotación laboral en la agricultura familiar del norte del Ecuador. En ese escenario, los resultados descriptivos de la tabla Io expresan que la población es predominantemente masculina, y que tiene en promedio la edad de i6,3 años. Asimismo, se evidencia una diferencia en el tamaño del hogar entre los hogares de los estudiantes de las regiones de la Costa y la Sierra.

TABLA 10

Principales Características Demográficas

\begin{tabular}{l|r|r|r|r}
\hline \multirow{2}{*}{ Región } & \multicolumn{2}{|c|}{ Género } & \multicolumn{1}{c|}{$\begin{array}{c}\text { Edad } \\
\text { (años) }\end{array}$} & \multicolumn{1}{c}{$\begin{array}{c}\text { Tamaño } \\
\text { del hogar }\end{array}$} \\
\cline { 2 - 3 } & \multicolumn{1}{|c|}{$\mathbf{M}$} & \multicolumn{1}{c|}{$\mathbf{F}$} & & \\
\hline Sierra & 60,5 & 39,5 & 16,4 & 6,6 \\
Costa & 68,5 & 31,5 & 16,2 & 5,0 \\
Amazonía & 51,6 & 48,4 & 16,3 & 5,3 \\
\hline Promedio total & 62,8 & 37,2 & 16,3 & 5,6 \\
\hline
\end{tabular}

Fuente: elaboración propia

La existencia de explotación laboral e infantil en la agricultura, como puede ser concebida según la perspectiva de la juventud rural, se demuestran en la figura 5. Además, según dicha perspectiva, más de la mitad de los encuestados considera que existe explotación laboral e infantil en la agricultura. 


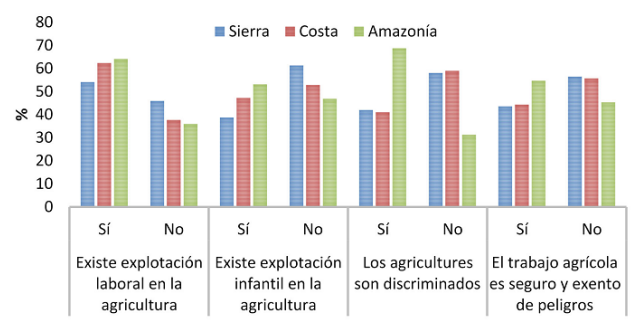

FIGURA 5

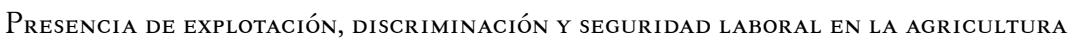

Nota: se presentan datos que contribuyen a la comprensión de las dimensiones de la seguridad social y los derechos de los trabajadores.

FUENTE: ELABORACIÓN PROPIA

Al explorar la participación de los jóvenes en la agricultura, se nota que un gran porcentaje de los encuestados de la región amazónica se ve atraído por la posibilidad de trabajar en la agricultura, y en similar proporción continuará con los estudios de agricultura en la universidad. En contraste, un gran porcentaje en la región de la Sierra no se inclina hacia por el trabajo agrícola familiar, y tampoco piensa continuar en el futuro con la formación profesional en agricultura. La figura 6 también evidencia que más de la mitad de los jóvenes piensa emigrar en un corto o mediano plazo. La principal motivación de los jóvenes de la Sierra y la Amazonía para la emigración es la finalidad de continuar con los estudios y la formación profesional en los centros urbanos, mientras que para la población joven de la Costa las motivaciones son buscar mejores oportunidades económicas y conseguir un buen empleo. En comparación, el porcentaje de las aspiraciones de emigrar es tres veces mayor en el grupo de jóvenes que en el conjunto de los agricultores descrito previamente.

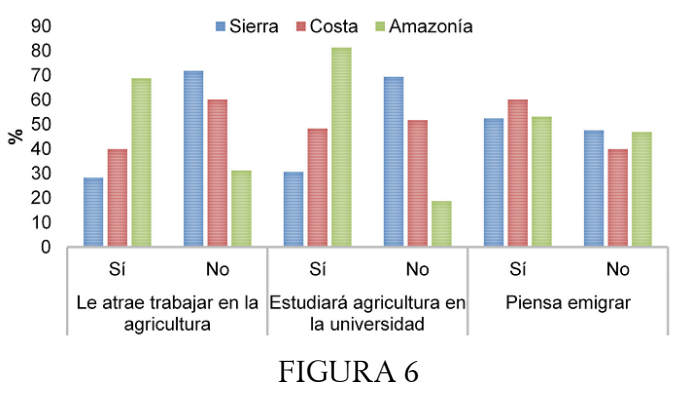

Percepciones de CONTINUIDAd y EMIGRACIÓN

Nota: se presentan datos que contribuyen a la comprensión de la continuidad de la agricultura y la migración de los jóvenes.

FuENTE: ElABORACIÓN PROPIA

Es destacable que los jóvenes consideran que la agricultura puede ser más atractiva si se mejoran las condiciones de vida de los hogares y si se incrementa la productividad agrícola (Figura 7). En la Amazonía, los jóvenes se muestran más atraídos hacia el trabajo en la agricultura y un gran porcentaje estudiará esa carrera en la universidad. Asimismo, este grupo considera que, para dar continuidad a la agricultura familiar en la región, se necesita apoyo técnico de parte de los gobiernos locales y nacionales.

$₫$ Sierra $₫$ Costa $₫$ Amazonía

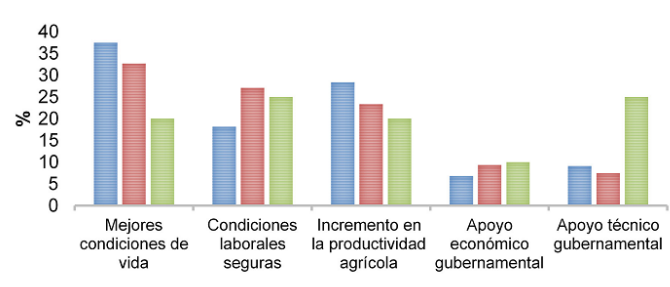

FIGURA 7

FACTORES A CONSIDERAR PARA EL INCREMENTO DEL INTERÉs EN LA AGRICULTURA

Nota: se presentan datos que contribuyen a la comprensión de las dimensiones de seguridad social y derechos de los trabajadores. FUENTE: ELABORACIÓN PROPIA 


\section{Conclusiones}

El uso de enfoques metodológicos mixtos cualicuantitativos en esta investigación demuestra que, aunada a las determinantes económicas, la falta de trabajo decente en la agricultura contribuye a diversos fenómenos. Con ello se hace referencia a (i) la migración; (ii) el empleo en actividades afuera de la finca; (iii) el cambio de la actividad económica principal, especialmente por actividades artesanales, y (iv) la reducción de las aspiraciones de los jóvenes en este sector a continuar con la agricultura familiar. Sin embargo, más de la mitad de la población encuestada considera que existe explotación laboral en la agricultura, lo cual se debe particularmente a los injustos salarios del sector. En ese escenario, la agricultura que se practica en la zona no es considerada una actividad económica segura y libre de peligros, y son pocos los cultivadores que cuentan con acceso al Seguro Social Campesino en las regiones de la Sierra y la Amazonía, y en mayor grado en la Costa.

Para su desarrollo y continuidad, la agricultura familiar depende netamente de la participación de los hijos y de otros miembros del hogar. En ese sentido, se ha demostrado que los agricultores que remuneran la mano de obra familiar, generan interés para que los hijos den continuidad a esa actividad, y terminan por incrementar la productividad laboral, al igual que el ingreso agrícola, eventualmente.

En tanto, la conjugación de las diversas problemáticas estudiadas influye la toma de decisiones para la emigración y el abandono de la agricultura; opciones que son consideradas por dos de cada diez agricultores. En ese escenario, los aspectos determinantes para el abandono de la agricultura son (i) la falta de apoyos económicos y técnicos al sector agrícola; (ii) los bajos ingresos agrícolas, y (iii) la baja productividad agrícola; mientras que en la Sierra es notoria (iv) la disminución de la fuerza laboral agrícola. Ante tal situación, la estrategia de los agricultores consiste en buscar empleo afuera de la granja, en donde el trabajo destacado es el de jornalero.

Asimismo, los resultados de la regresión logística son significativos, y mediante la interpretación de los coeficientes, esta investigación ofrece resultados representativos. Se determina que, a nivel de la agricultura familiar, las variables que aumentan la decisión de emigrar en los agricultores son el (i) incremento de la edad de los agricultores, (ii) los bajos niveles de educación, (iii) el tamaño del hogar, (iv) la tenencia de la tierra, (v) la baja rentabilidad agrícola, (vi) la poca participación de los hijos, (vii) la contribución de otros miembros del hogar en las actividades agrícolas, (viii) la predominancia de la explotación laboral, y (ix) el abandono de la agricultura.

Adicionalmente, las condiciones laborales de la agricultura familiar del norte del Ecuador no están alineadas con los pillares del trabajo decente, ni son contempladas en su agenda, de acuerdo con el análisis realizado a cada una los indicadores que componen sus dimensiones. Particularmente, en la región de la Sierra (Otavalo-Imbabura) se presentan mayores procesos de abandono de la agricultura, emigración y desagrarización; además, el cultivo se caracteriza por ser minifundista ( 0,327 ha en promedio), contando con ingresos económicos extremadamente bajos en algunos casos, y en otros sin generar ningún ingreso, de modo que no se puede remunerar la mano de obra familiar: una circunstancia que ocasiona una baja participación por parte de hijos y de otros miembros del hogar, a la vez que fomenta la explotación laboral agrícola e infantil. En este lugar de estudio, la producción agrícola familiar se ha convertido en una actividad complementaria del autoconsumo y la subsistencia del hogar; una función que está siendo relevada por el desarrollo manufacturero y turístico del sector.

Mientras tanto, en la región de la Costa existe un mayor abandono de la actividad agrícola; sin embargo, existe un menor porcentaje de agricultores que piensa emigrar. Particularmente, en lo que atañe al enfoque de los jóvenes, cabe anotar que más de la mitad piensa emigrar. En contraste, al existir una mayor participación y remuneración de la mano de obra familiar en la Amazonía, en tal caso los recursos naturales - consistentes en el tamaño de la UPA-, las posibilidades de la emigración y el abandono de la agricultura son menores. 
En lo que atañe a la perspectiva de la juventud rural, más de la mitad considera que hay explotación laboral e infantil en la agricultura, y además arguye que esta no es una actividad segura, ni está exenta de peligros. Según lo anterior, los factores descritos acarrean (i) menor interés por esta actividad económica, (ii) una mayor migración del campo a la ciudad, y (iii) mayor abandono de las actividades agrícolas. En ese sentido, los jóvenes encuestados en la Amazonía demuestran tener un mayor interés por trabajar y estudiar la agricultura; además, la tendencia a la migración es menor en esta zona, a pesar de que las condiciones socioeconómicas y laborales del sector agrario sean las más bajas, a comparación de los demás sitios sujetos a estudio.

En el contexto descrito, es destacable que la idea de emigrar sea común entre los jóvenes de los tres sitios de estudio. Lo anterior demuestra que se necesita actuar mediante el mejoramiento de la calidad del trabajo agrícola y la productividad agrícola; factor que se traduce en el incremento económico.

En suma, el análisis de las dinámicas dadas a nivel de la agricultura familiar, y de la juventud rural, permite establecer que el fenómeno de la migración, al igual que el abandono de la actividad económica principal -ambos aspectos que se derivan por igual de las precarias condiciones laborales ocasionadas por la pobreza-, afectan al desarrollo agrícola y rural; son factores que establecen, de ese modo, un círculo vicioso. Además, permite establecer un escenario de desagrarización del campo y de acabamiento de la agricultura familiar, de modo que se pone en riesgo la seguridad alimentaria local y regional. De esta manera, se busca repercutir en el enfoque de los proyectos y políticas de desarrollo agrícola-rural con la finalidad de que la articulación del trabajo decente se perfile como un eje transversal.

Ante el panorama expuesto, es importante reconocer que diversos estudios conducidos en Malawi, Tanzania y Zanzíbar determinan que, para materializar las aspiraciones de la población rural joven, es necesario dar acceso al trabajo decente y productivo. En esos países, las actividades implementadas de acuerdo con el marco del trabajo decente han permitido que las poblaciones jóvenes intervengan en nuevas oportunidades agroempresariales; que tengan una mayor participación social comunitaria, y que se incrementen sus ingresos hasta en un 60\% (FAO, 20r4b). Otros estudios de caso también demuestran que la implementación del trabajo decente (i) potencialmente incrementa y mejora la productividad laboral y (ii) estabiliza los salarios; por lo tanto, (iii) incrementa las ventas de las industrias locales (Pyke, 20I4). En consecuencia, parte de la generación de desarrollo equitativo sustentable y democrático se basa en el impulso de políticas institucionales dirigidas hacia el trabajo decente, los derechos laborales y el fomento de empleo digno (Gálvez et al., 20I6; Ghai, 2003; OIT, 20I2). Este trabajo sustenta la implementación del trabajo decente como alternativa para el desarrollo rural, y contribuye con información primaria a la creación de conocimiento sobre una temática poco explorada, así como al diseño de políticas coherentes.

\section{Implicaciones políticas}

Los resultados obtenidos en esta investigación contribuyen a determinar algunas de las variables que influyen en la multicausalidad de la pobreza, la migración y el desarrollo. Para la reducción de la pobreza y la migración, se deben diseñar y orientar las políticas de desarrollo rural actuales, con la finalidad de que se considere el mejoramiento de las condiciones laborales agrícolas como una alternativa para la productividad, el crecimiento económico y la generación del desarrollo humano y sostenible. En concordancia, se puede empezar por atacar las determinantes identificadas en esta investigación, con los fines de mejorar el ingreso agrícola, estimular la partición de la familia en la agricultura, reducir la explotación laboral y disminuir la migración de los espacios rurales a los urbanos. Según lo expuesto, los lineamientos para el marco de trabajo están compuestos de tres factores esenciales: (i) la educación, idónea en particular para actualizar el conocimiento sobre prácticas agrícolas eficientes en la agricultura familiar, en el marco de la agricultura climáticamente inteligente (CSA, por sus siglas en inglés), y para atender 
el desconocimiento de las condiciones laborales adecuadas o el trabajo decente de los trabajadores. Asimismo, es necesario (ii) el financiamiento del sector rural y la agricultura familiar, para la generación de empleo rural productivo digno; y (iii) la innovación en las áreas del desarrollo y el apoyo tecnológico necesarios para la agricultura familiar y el sector agrícola en general.

\section{Referencias}

Banco Mundial. (2008). Informe de desarrollo mundial 2008. Agricultura para el desarrollo. Washington, DC: Banco Mundial.

Barrientos, S. (2007). Global production systems and decent work (Working Paper N.o 77). Ginebra: International Labour Organization.

Bernstein, H. (2012). Dinámicas de clase y transformación agraria. Zacatecas: Universidad Autónoma de ZacatecasPorrúa.

Bilsborrow, B. (2002). Migration, population change, and the rural environment. Environment Change and Security Program, I4(9), 69-94.

Brassel, F., Ruiz, P. y Zapata, A. (2008). La estructura agraria en el Ecuador: una aproximación a su problemática y tendencias. En F. Brassel, S. Herrera y M. Laforge (eds.), ¿̇Reforma agraria en el Ecuador?: viejos temas, nuevos argumentos (pp. 17-30). Quito: Sistema de Investigación de la Problemática Agraria en el Ecuador.

Carmagnani, M. (2008). La agricultura familiar en América Latina. Problemas del Desarrollo. Revista Latinoamericana de Economía, 39(153), II-56.

Food and Agriculture Organization. (2012). Decent rural employment for food security: A case for action. Roma: Food and Agriculture Organization.

Food and Agriculture Organization. (2014a). Turning family farm activity into decent work. Roma: Food and Agriculture Organization.

Food and Agriculture Organization. (2014b). Private and public partnership model for youth employment in agriculture experiences from Malawi, Tanzania Mainland and Zanzibar archipelago (Rural Employment Case Study Series N.o 4). Roma: Food and Agriculture Organization.

Food and Agriculture Organization. (2016). The estate of food and agriculture. Climate change, agriculture and food security. Roma: Food and Agriculture Organization.

Filmer, D. y Fox, L. (2014). Youth employment in sub-Sabaran Africa: Agricultural career paths for the future (Africa Development Series). Washington, DC: The World Bank.

Gálvez, S., Gutiérrez, E., Picazzo, E. y Osorio, J. (2016). El trabajo decente, una alternativa para reducir la desigualdad en la globalización: el caso de México. Región y Sociedad, 28(66), 56-94.

Garner, E. y O'Campos, A. (20I4). Identifying the "family farm": an informal discussion of the concepts and definitions (ESA Working Paper N.o I4-Io). Italia: Food and Agriculture Organization.

Ghai, D. (2003). Decent work: Concept and indicators. International Labour Review, I42(2), II3-I46.

Gray, C. y Bilsborrow, R. (2014). Consequences of out-migration for land use in rural Ecuador. Land Use Policy, 36(2014), I82-191

Gray, C. (2009). Environment, land, and rural out-migration in the Southern Ecuadorian Andes. World Development, $37(2), 457-468$.

Hertel, S. (2009). Human rights and the global economy: Bringing labor rights back in. Maryland Journal of International Law, 24(I), 240-252. 
Instituto Ecuatoriano de la Seguridad Social. (2006). Seguro Campesino. Recuperado de https://www.iess.gob.ec/e s/19

Instituto Nacional de Estadísticas y Censos. (2014). Encuesta Nacional de Empleo, Desempleo y Subempleo. Quito: Instituto Nacional de Estadísticas y Censos.

Instituto Nacional de Estadísticas y Censos. (2016). Encuesta Nacional de Empleo, Desempleo y Subempleo. Quito: Instituto Nacional de Estadísticas y Censos. Recuperado de http://www.ecuadorencifras.gob.ec/document os/web-inec/EMPLEO/2016/Junio-2016/Presentacion_Empleo_Juniozor6.pdf

Jokisch, D. (2002). Migration and agricultural change: The case of smallholder agriculture in Highland Ecuador. Human Ecology, 30(4), 523-550.

Kaenzig, R. y Piguet, E. (2016). Migration and climate change in Latin America and the Caribbean. En E. Piguet y F. Laczko (eds.), People on the move in a changing climate (pp. 155-176). Ginebra: Springer.

Kucera, D. (200I). The effects of core worker rights on labour costs and foreign direct investment: Evaluating the "conventional wisdom". Ginebra: International Institute of Labour Studies.

Organización Internacional del Trabajo. (junio, 1999). Conferencia Internacional del Trabajo: Trabajo decente. Ginebra: Organización Internacional del Trabajo. Recuperado de http://www.ilo.org/public/spanish/standards/rel m/ilc/ilc87/rep-i.htm

Organización Internacional del Trabajo. (mayo, 2006). Decent work in the Americas: An agenda for the Hemisphere, 2006-2015. Brasilia: Organización Internacional del Trabajo.

Organización Internacional del Trabajo. (2007). The ILO at a glance. Ginebra: Organización Internacional del Trabajo.

Organización Internacional del Trabajo. (2012). Working towards sustainable development: Opportunities for decent work and social inclusion in a green economy. Ginebra: Organización Internacional del Trabajo.

Organización Internacional del Trabajo. (2016). Global estimates of modern slavery: Forced labour and forced marriage. Ginebra: Organización Internacional del Trabajo.

Organización Internacional del Trabajo. (2017). Global estimates of child labour: Results and trends, 2012-2016. Ginebra: Organización Internacional del Trabajo.

Organización de las Naciones Unidas. (2013). The number of international migrants worldwide reaches 232 million (Population Facts N.o 2). Recuperado de https://esa.un.org/unmigration/documents/the_number_of_inter national_migrants.pdf

Organización de las Naciones Unidas. (2016). Progress towards the Sustainable Development Goals. Recuperado de http s://unstats.un.org/sdgs/files/report/2016/secretary-general-sdg-report-2016--EN.pdf

Programa Mundial de Alimentación. (2017). From ideas to actions: Supporting local government capacities to improve food and nutrition security in Ecuador. Quito: Programa Mundial de Alimentación.

Pyke, F. (20I4). Promoting cluster development through decent work: The case of the Surat diamond processing cluster. En K. Das (ed.), Globalization and standards. India studies in business and economics globalization and standards (pp. 209-223). Nueva Delhi: Springer.

Sili, M., Fachelli, S. y Meiller, A. (2017). Juventud rural: factores que influyen en el desarrollo de la actividad agropecuaria. Reflexiones sobre el caso argentino. Revista de Economía y Sociología Rural, 54(4), 635-652.

Sen, A. (1999). Development as freedom. Oxford: Oxford University Press.

Secretaría Nacional de Planificación y Desarrollo. (2013). Plan Nacional para el Buen Vivir 2013-2017. Quito: Senplades. 
Taylor, J. (1999). The new economics of labour migration and the role of remittances in the migration process. International Migration, 37(I), 63-88.

Van Vliet, J., Schut, A., Reidsma, P., Descheemaeker, K., Slingerland, M., Van de Ven, K. W. y Giller, K. E. (2015). De-mystifying family farming: Features, diversity and trends across the globe. Global food security, $5(2015), \mathrm{II}-\mathrm{I} 8$.

Viteri, J. (2010). Trabajo decente: diagnóstico nacional del Ecuador. Quito: Plades.

Wiggins, S. (2016). Agricultural and rural development reconsidered (IFAD Research Series N.o I). Roma: International Fund for Agricultural Development.

\section{Notas}

* Artículo de investigación.

Licencia Creative Commons BY-4.० 